\title{
I. Der Werdegang Gerhard Schröders bis zu seinem Amtsantritt als Außenminister
}

Am 31. Dezember 1989 verstarb Gerhard Schröder in seinem Ferienhaus Atterdag in Kampen auf Sylt. Seine politische Karriere endete neun Jahre zuvor, als er sich entschloß, in seinem Wahlkreis Düsseldorf-Mettmann nicht mehr für die Bundestagswahl 1980 zu kandidieren, weil ihm die CDU einen vorderen Platz auf der Landesliste zur Absicherung seiner Kandidatur verweigerte ${ }^{1}$. Seine letzten Lebensjahre verbrachte er weitgehend abgeschieden im Kreis seiner Familie in Bonn. Aus der Tagespolitik hatte er sich beinahe vollständig zurückgezogen. Nur in der Presse waren hin und wieder noch Kommentare und Artikel von ihm zu lesen. Größere Beachtung fand eine sechsteilige Serie von persönlichen Erinnerungen an Adenauer, Erhard, Kennedy und de Gaulle, die 1988 im Rheinischen Merkur erschien $^{2}$. Im selben Jahr hatte er noch einen großen Fernsehauftritt, als er dem ZDF für die Reihe Zeugen des Jabrbunderts Rede und Antwort stand ${ }^{3}$. Nach seinem Tod erinnerte die Bundesrepublik Deutschland in einem Staatsakt am 12. Januar 1990 im Bundestag an seine Verdienste. Bundeskanzler Helmut Kohl und Bundestagspräsidentin Rita Süssmuth bezeichneten in ihren Ansprachen den früheren Innen-, Außen- und Verteidigungsminister als eine der prägenden politischen Figuren der Bundesrepublik ${ }^{4}$. Über alle Parteigrenzen hinweg werden Schröders Leistungen anerkannt: So gehört er für Hans-Dietrich Genscher vor allem wegen seiner außenpolitischen Leistungen „zu den großen Persönlichkeiten der deutschen Nachkriegspolitik" 5 . Auch der frühere Bundeskanzler Helmut Schmidt würdigte Schröder noch zu dessen Lebzeiten ${ }^{6}$. Doch trotz seiner Bedeutung für die politische Entwicklung der Bundesrepublik nahm die Öffentlichkeit vom Tod eines Mannes, der das politische Leben für Jahrzehnte entscheidend mitbestimmt hatte, kaum Notiz. „Für die jüngere Generation ist Gerhard Schröder kaum noch

1 Vgl. dazu Adelbert SCHRÖDER, Mein Bruder Gerhard Schröder, S. 147, sowie den Artikel von Herbert Riehl-Heyse: „Staatsmännisch - auch mit zusammengebissenen Zähnen“, in: SÜDDEUTSCHE ZEITUNG vom 11. 9. 1980, S. 3.

2 SCHRÖDER, Goldenes Firmenschild wollte Erhard nicht sein; ders., Der Wahltriumph rettete den Kanzler nicht; ders., „Donnerwetter, das kann ja fein werden ...“; ders., Eine Schlacht um Otto John; ders., "Je weiter östlich, desto besser"; ders., Mit dem „Alten“ gebaut und gelitten; ders., Mein Bild von de Gaulle; ders., Ein Stück Geschichte, das uns bleiben wird.

3 Das Interview wurde auch in gedruckter Form veröffentlicht: SCHRÖDER, Im Gespräch mit Meinhold Krauss.

4 Vgl. Bulletin 1990, S. 41-44.

5 GENSCHER, Erinnerungen, S. 107.

6 SCHMIDT, Schablonen, S. $11 \mathrm{f}$. Dieser Aufsatz erschien in einer Festschrift, die 1980 anläßlich von Schröders 70. Geburtstag herausgegeben wurde: KUNST (Hrsg.), Dem Staate verpflichtet. Darin würdigen u. a. auch Hans-Dietrich Genscher (S. 85-90) und der damalige Bundespräsident Karl Carstens (S. 7-9) Schröders politisches Lebenswerk. 
ein Begriff“, beklagte Klaus Dreher in der Süddeutschen Zeitung7. In der Presse erschienen nur einige knapp gehaltene Nachrufe ${ }^{8}$. Mit dem Echo auf den Tod von Franz Josef Strauß, Schröders langjährigen politischen Rivalen, war die öffentliche Reaktion auf sein Ableben nicht vergleichbar.

Das geringe Interesse an seiner Person ist schwerlich nachzuvollziehen. Immerhin war Schröder 16 Jahre lang Bundesminister und galt in den sechziger Jahren als einer der ersten Anwärter auf die Kanzlerschaft, die ihm jedoch verwehrt blieb. 1963 verzichtete er in Kenntnis der Stimmungslage in seiner Partei auf eine Kandidatur gegen Ludwig Erhard ${ }^{9}$. Drei Jahre später trat er gegen Barzel, Kiesinger und Hallstein um die Nachfolge Erhards an und verlor in einer Stichwahl der CDU/CSU-Fraktion gegen den Ministerpräsidenten von Baden-Württemberg, Kurt Georg Kiesinger ${ }^{10} .1969$ bewarb er sich um das Amt des Bundespräsidenten, unterlag aber in der Bundesversammlung Gustav Heinemann. Es fehlten ihm sechs Stimmen ${ }^{11}$. In den siebziger Jahren gelang es ihm nicht mehr, sich in der ersten Reihe der politischen Bühne zu halten. Sein Versuch 1971, die Kanzlerkandidatur zu erreichen, scheiterte ebenso wie seine Bewerbung zwei Jahre später um den Vorsitz der CDU/CSU-Fraktion im Bundestag12. Als Vorsitzender des Auswärtigen Ausschusses des Bundestags von 1969 bis 1980 wirkte er aber weiterhin an der Gestaltung der Außenpolitik der Bundesrepublik mit. Aufsehen erregte seine Reise in die Volksrepublik China im Juli 1972. Sein Gespräch mit Ministerpräsident Chou En-lai bereitete den Boden für die Aufnahme diplomatischer Beziehungen mit Peking ${ }^{13}$. Es mag erstaunen, daß er im Rückblick in dieser Mission obne Auftrag seinen wichtigsten Beitrag zur auswärtigen Politik Bonns sah ${ }^{14}$. Einen heftigen Streit in der Union löste er aus, als er unmittelbar nach einem Besuch in Israel im Dezember 1974 spontan in Damaskus als erster Politiker aus der Bundesrepublik mit dem Chef der PLO, Jasir Arafat, zusammentraf ${ }^{15}$.

Der mangelnde Erfolg bei allen seinen Versuchen ab Mitte der sechziger Jahre, ein von ihm angestrebtes Amt zu erreichen, gibt Schröders Lebenslauf beinahe eine tragische Note ${ }^{16}$. Sicherlich war sein Charakter ein Hemmschuh. Weder in

7 Vgl. den Artikel von Klaus Dreher: „Politiker mit analytischem Verstand“, in: SÜDDEUTSCHE ZEITUNG vom 3. 1. 1990, S. 5.

8 Vgl. z.B. die Artikel „Einer der profiliertesten Politiker. Zum Tod von Bundesminister a.D. Gerhard Schröder“, in: DAS PARLAMENT vom 5. 1. 1990, S. 5; „Der Wortführer der Atlantiker eröffnete neue Wege in der Außenpolitik“, in: FRANKFURTER ALLGEMEINE ZEITUNG vom 3.1. 1990, S. 4; sowie die Artikel von Herbert Kremp: „Am Jahreswechsel starb Gerhard Schröder“, in: DIE WELT vom 3. 1. 1990, S. 4, und von Klaus Dreher: „Politiker mit analytischem Verstand“, in: SÜDDEUTSCHE ZEITUNG vom 3. 1. 1990, S. 5.

9 Vgl. dazu Adelbert SCHRÖDER, Mein Bruder Gerhard Schröder, S. 134.

10 Offiziell waren für die Nachfolge von der CDU/CSU-Fraktion Barzel, Gerstenmaier, Kiesinger und Schröder nominiert. Gerstenmaier verzichtete jedoch. Vgl. dazu ADG 1966, S. 12801.

11 Vgl. KleinmanN, Geschichte der CDU, S. 306.

12 Vgl. ebenda, S. 324 und S. 351; CARSTENS, Erinnerungen, S. 423 f.

13 Vgl. dazu die Erinnerungen Schröders und seiner Frau Brigitte über diese Reise: SCHRÖ-

DER, Mission ohne Auftrag.

14 Vgl. Tiesenhausen, Die Sache mit China, S. 30.

15 Vgl. Adelbert SCHRÖDER, Mein Bruder Gerhard Schröder, S. $144 \mathrm{f}$.

16 Vgl. OSTERHELD, Außenpolitik, S. 246. 
seiner Partei noch in der Bevölkerung genoß er große Popularität. Schröder war zwar angesehen, aber kein beliebter, weil volkstümlicher Politiker. Er selbst glaubte, seine Bescheidenheit und seine zurückhaltende Art hätten einen erfolgreicheren Verlauf seiner politischen Karriere verhindert. „Daß ich nicht genug Reklame habe machen können für mich“, sei sein größter Fehler gewesen, meinte er 1980 gegenüber Herbert Riehl-Heyse anläßlich seines Rückzugs vom aktiven politischen Leben ${ }^{17}$.

Es ist jedoch unbestritten, daß Schröder über die notwendigen Fähigkeiten eines herausragenden Politikers verfügte. Hans-Peter Schwarz sieht in ihm ein „politisches Naturtalent" ${ }^{18}$. Er galt als fleißig, tüchtig, klug, intelligent, diszipliniert und geschickt, dachte in erster Linie pragmatisch und besaß das wichtige Gespür für das politisch Machbare ${ }^{19}$. Dies war nicht nur die übereinstimmende Einschätzung von Journalisten und Zeithistorikern, sondern auch die seiner Mitarbeiter und ausländischen Kollegen. Für den späteren Bundespräsidenten Karl Carstens, der Schröder im Auswärtigen Amt und im Verteidigungsministerium als Staatssekretär diente, war dessen Stärke „sein analytisch geschulter und unbestechlicher Verstand" 20 . Carstens rühmt vor allem die vorbildhafte Personalpolitik seines früheren Vorgesetzten. Sie sei nie parteipolitisch gefärbt und stets vorurteilsfrei gewesen ${ }^{21}$. Menschen, die näheren Kontakt mit ihm hatten, beschreiben ihn als freundlich, zuverlässig und angenehm im Umgang 22 . Zudem war Schröder auch eine Kämpfernatur: Durch Rückschläge ließ er sich nicht unterkriegen ${ }^{23}$. Er war durchaus für das Amt des Bundeskanzlers befähigt. Sein Ehrgeiz und der Drang nach Macht, der ihm nachgesagt wurde ${ }^{24}$, standen einer derartigen Entwicklung seiner Karriere nicht entgegen, denn dies waren Eigenschaften, die für einen Spitzenpolitiker unabdingbar sind. Horst Osterheld, der als Leiter des Außenpolitischen Büros im Bundeskanzleramt zwischen 1961 und 1969 sehr eng mit Schröder zusammenarbeitete, kritisierte an ihm jedoch, er habe Entscheidungen manchmal zu rasch und zu wenig unvoreingenommen getroffen. Auch habe es ihm an Selbstkritik gemangelt ${ }^{25}$.

Schröders größtes Manko war sicherlich, daß er über kein gewinnendes Wesen verfügte. Es fehlte ihm der Sinn für Liebenswürdigkeit. Gegenüber den meisten Menschen verhielt er sich zurückhaltend und distanziert ${ }^{26}$. Selbst bei Personen, die häufig Umgang mit ihm hatten, erweckte er den Eindruck, daß er keine $\mathrm{Ge}$ -

17 Vgl. den Artikel von Herbert Riehl-Heyse: „Staatsmännisch - auch mit zusammengebissenen Zähnen“, in: SÜDDEUTSCHE ZEITUNG vom 11. 9. 1980, S. 3.

18 SCHWARZ, Adenauer II, S. 357.

19 Vgl. dazu die Charakterisierungen in HenkEls, 111 Bonner Köpfe, S. 291; SCHWARZKoPf/ Wrangel, Chancen, S. 146 f.; WAGNer, Der neue Mann im Außenamt, S. 27-31.

20 CARSTENS, Erinnerungen, S. 234. Dies bekräftigen BlankENHORN, Verständnis, S. 290 und S. 458; OSTERHELD, Außenpolitik, S. $245 \mathrm{f}$.

$21 \mathrm{Vgl}$. CARSTENS, Erinnerungen, S. 234. Ebenso äußert sich Schröders langjähriger Persönlicher Referent, Klaus Simon (ZEITZEUGENBEFRAGUNG Simon).

22 Vgl. McGhee, Botschafter, S. 46; ZeITZEUGenbefragung Simon.

23 Vgl. OSTERHELD, Außenpolitik, S. 245; WagneR, Der neue Mann im Außenamt, S. 30.

24 Vgl. OSterheld, Außenpolitik, S. 245 f. und S. 309; LAHR, Zeuge, S. 348.

25 Vgl. OSTERHELD, Außenpolitik, S. $245 \mathrm{f}$.

26 Vgl. Wagner, Der neue Mann im Außenamt, S. 29. 
fühle kenne ${ }^{27}$. Schröders unterkühltes Auftreten, sein ausgeprägtes Selbstbewußtsein und seine Vorliebe für elegante Kleidung trugen ihm den Ruf der Arroganz, Überheblichkeit, Eitelkeit und Hochmütigkeit ein ${ }^{28}$. Auf seine Mitmenschen machte er den Eindruck, als halte er sich für intellektuell überlegen. Es heißt, Schröder habe nur dann Emotionen gezeigt, wenn er auf Widerspruch stieß oder mit den Handlungen von Mitarbeitern unzufrieden war. In einem solchen Fall konnte er auch mit einem Wutausbruch reagieren ${ }^{29}$, obwohl er ansonsten über sehr große Selbstbeherrschung verfügte ${ }^{30}$. Rolf Lahr, neben Carstens zweiter Staatssekretär im Auswärtigen Amt, bezeichnete den Umgang mit ihm als „schwierig“31. Kein Wunder also, daß Schröder Probleme hatte, Menschen für sich zu gewinnen. Auch in der Bevölkerung konnte er auf Grund seiner Persönlichkeit kaum Gefühle für sich wecken ${ }^{32}$. Sein kühles Wesen führte dazu, daß $\mathrm{ihm}$ nur wenig Sympathien entgegengebracht wurden. Die Ablehnung, die Schröder hervorrief, reichte soweit, daß ihm sein Verhalten oft negativ ausgelegt wurde. Seine pragmatische Art wurde als Opportunismus interpretiert und es wurde ihm nachgesagt, er sei berechnend ${ }^{33}$. Sein Machthunger, hieß es, sei größer als seine Überzeugungen. So sahen manche in seiner Tätigkeit als Vorsitzender des Evangelischen Arbeitskreises der CDU/CSU nicht so sehr ein Engagement aus christlicher Überzeugung, sondern den Versuch, sich in der Union eine Hausmacht zu schaffen ${ }^{34}$. Schröder wurde als "vollendeter Eisberg " 35 und "menschlicher Kältestrahler" beschrieben, der den "Charme eines Elektronengehirns" habe ${ }^{36}$. Er sei „glatt und kalt und zu ,preußisch““37.

Diese Charakterisierungen werden zumindest dem Privatmenschen Gerhard Schröder nicht gerecht. Außerhalb der Politik konnte er eine ganz andere Seite seines Wesens zeigen: Hier war er "redselig“ und „gesellig "38, „offen und sogar herzlich"39. Auch Liebenswürdigkeit, die in der Öffentlichkeit kaum zum Vorschein trat, zeigte er privat ${ }^{40}$. Seine vielfältigen Interessen sind kaum bekannt: Schröder hatte ein Faible für moderne Kunst und war auf diesem Gebiet ein eifriger Sammler. Er liebte klassische Musik, vor allem Richard Wagner. Als begeisterter Fotograf war er über Jahrzehnte Vorsitzender der Deutschen Gesellschaft für Fotografie. Seine Leidenschaft galt auch theologischen Problemen. Hier schätzte

27 Vgl. PoppINGA, „Das Wichtigste ist der Mut“, S. 338.

28 Vgl. KRONE, Tagebücher, Eintrag vom 9. 10. 1954, S. 146; OSTERHELD, Außenpolitik, S. $245 \mathrm{f}$. Zu Schröders Vorliebe für teuere Kleidung seit seiner Studentenzeit, vgl. auch Adelbert SCHRÖDER, Mein Bruder Gerhard Schröder, S. $67 \mathrm{f}$.

29 Vgl. SAHM, Diplomaten, S. 182.

30 Vgl. Carstens, Erinnerungen, S. 350.

31 LAHR, Zeuge, S. 348.

32 Vgl. SCHWARZKOPF/Wrangel, Chancen, S. 147.

33 Vgl. WaGneR, Der neue Mann im Außenamt, S. 27-29.

34 Vgl. SAHM, Diplomaten, S. 182; PFETSCH, Außenpolitik, S. 85.

35 Henkels, 111 Bonner Köpfe, S. 290.

36 WAGNER, Der neue Mann im Außenamt, S. 29.

37 KRONE, Tagebücher, Eintrag vom 26. 4. 1959, S. 352.

38 WAGNER, Der neue Mann im Außenamt, S. 31.

39 SCHWARZKOPF/Wrangel, Chancen, S. 150.

40 Vgl. Diehl, Zwischen Politik und Presse, S. 26; LAHr, Zeuge, S. 362 f. 
er besonders den Schweizer Theologen Karl Barth. Mit der Philosophie Immanuel Kants beschäftigte er sich sein Leben lang ${ }^{41}$. Doch über seine persönlichen $\mathrm{Ge}-$ danken und Gefühle hat Schröder so gut wie nichts nach außen dringen lassen. Nach eigenem Bekenntnis verabscheute er es, über sich selbst zu sprechen ${ }^{42}$. So offenbarte er auch in seinem Interview für die ZDF-Sendung Zeugen des Jabrbunderts, ein Jahr vor seinem Tod, wenig Privates ${ }^{43}$. Sicherlich haben Schröders Erfahrungen in der Zeit des Nationalsozialismus diesen Charakterzug gefördert. Zwölf Jahre lang war er gezwungen, seine Abneigung gegen das NS-Regime zu verbergen. Welchen Einschnitt die „Machtübernahme“ Hitlers 1933 für den damals 23-jährigen und seine Lebensplanung darstellte, ist aus heutiger Sicht nur schwer nachzuempfinden:

Schröder entstammte einer kleinbürgerlichen, evangelischen Familie aus Friesland. Sein Vater, Jan Schröder, ein fleißiger und ehrgeiziger Mann, hatte den beruflichen und gesellschaftlichen Aufstieg "vom Schiffsjungen zum Reichsbahnrat" geschafft ${ }^{44}$. Als Bahnbeamter wurde er häufig versetzt, wodurch Gerhard Schröder nicht in der friesischen Heimat seiner Eltern, Jan und Anita, zur Welt kam, sondern im Saargebiet. Am 11. September 1910 wurde er in Saarbrücken, wo sein Vater seit 1909 als Eisenbahn-Assistent tätig war, geboren. In seine Kindheit fiel der Erste Weltkrieg. Im September 1915 wurde der Vater zur Armee eingezogen, so daß die Mutter den jungen Gerhard und die 1913 geborene Schwester MarieRenate - sein Bruder Adelbert wurde erst 1921 geboren - bis zum Ende des Krieges allein erziehen mußte. Noch als Kind erfuhr Schröder somit, welche Auswirkungen die Politik auf das Schicksal des einzelnen Menschen haben kann. Auch die Nachkriegszeit trug dazu bei, diese Erkenntnis zu vertiefen. Laut den Bestimmungen des Versailler Vertrages wurden das Saargebiet und das Rheinland von französischen Truppen besetzt. Die Schröders gerieten in Konflikt mit der Besatzungsmacht. Im Sommer 1921, als Gerhard die erste Klasse des Gymnasiums in Saarbrücken besuchte, wurde die Familie aus dem Saargebiet ausgewiesen. Zunächst fanden die Schröders Zuflucht in Trier. Doch bereits eineinhalb Jahre später wurden sie erneut vertrieben, da ihre Wohnung in der Klosterstraße in einem Stadtviertel lag, das zur Unterbringung französischer Soldatenfamilien requiriert worden war. Bei einer fremden Familie wurden sie zur Notunterkunft einquartiert. Im Mai 1923 verhafteten die französischen Behörden den Vater, weil er an der Organisation des passiven Widerstandes gegen die französische Ruhrbesetzung beteiligt war und wiesen ihn zusammen mit seiner Familie auch aus dem Rheinland aus. Erst im November 1924 konnten die Schröders in ihre alte

\footnotetext{
41 Vgl. dazu Adelbert SCHRÖDER, Mein Bruder Gerhard Schröder, S. 50; SCHRÖDER, Im Gespräch mit Meinhold Krauss, S. 19f.; ZeITZEUGENBEFRAGUNG Simon.

42 Vgl. den Artikel von Herbert Riehl-Heyse: „Staatsmännisch - auch mit zusammengebissenen Zähnen“, in: SüDDEUTSCHE ZEITUNG vom 11. 9. 1980, S. 3. Deshalb zögerte Schröder auch, seine Memoiren zu schreiben: „Das liegt mir eigentlich gar nicht“ (ebenda).

$43 \mathrm{Vgl}$. SCHRÖDER, Im Gespräch mit Meinhold Krauss.

44 Adelbert SCHRÖDER, Mein Bruder Gerhard Schröder, S. 55.
} 
Wohnung nach Trier zurückkehren; bis zu diesem Zeitpunkt lebten sie im rechtsrheinischen Hessen: zunächst in Friedberg, anschließend in Gießen ${ }^{45}$.

Bereits als 15-jähriger betätigte sich Gerhard Schröder zum ersten Mal politisch. Bei den Reichspräsidentenwahlen 1925 klebte er Wahlplakate für die DNVP, die die Kandidatur Hindenburgs unterstützte ${ }^{46}$. Prägend für die politischen Ansichten des Jugendlichen war jedoch sein Vater. Dieser war trotz seiner nationalen Gesinnung ein Anhänger der überwiegend republiktragenden Deutschen Volkspartei Gustav Stresemanns. Obwohl er und seine Familie persönlich schwer unter der französischen Besatzung gelitten hatten, unterstützte er dennoch aus Gründen der politischen Vernunft die Verständigungspolitik Stresemanns mit Frankreich ${ }^{47}$. Er sah die Notwendigkeit einer solchen Politik ein, die für die $\mathrm{Zu}$ kunft ein friedliches Zusammenleben der rheinischen Nachbarn garantieren sollte. Diese Einsicht ging jedoch verständlicherweise nicht soweit, daß aus den Schröders nach den Erlebnissen der letzten Jahre plötzlich glühende Verehrer der französischen Politik wurden. Als Gerhard Schröder 35 Jahre später Außenminister geworden war, fühlte sich mancher politischer Beobachter bemüßigt, dessen Abneigung gegen die Politik de Gaulles mit den schlechten Erfahrungen zu erklären, die Schröder als Jugendlicher mit der französischen Besatzungspolitik gemacht hatte ${ }^{48}$. Gut in dieses Bild paßte dabei die Tatsache, daß de Gaulle in den zwanziger Jahren Bataillonskommandant in Trier war und unmittelbar neben dem humanistischen Gymnasium wohnte, das Schröder damals besuchte, so daß sich beide zuweilen auf dem Schulweg begegneten ${ }^{49}$.

Der berufliche Aufstieg des Vaters ermöglichte es Schröder, nach seinem Abitur im Frühjahr 1929 am Kaiser-Wilhelm-Gymnasium in Trier ein Studium zu beginnen. Schon seit längerem war seine Wahl auf die Rechtswissenschaften gefallen. Als Bewunderer Kants - und weil die Stadt weit von Trier entfernt war - schrieb er sich für das Sommersemester 1919 an der Universität Königsberg ein ${ }^{50}$. Danach folgte ein Auslandssemester in Edinburgh. Da es ihm in Schottland ausgesprochen gut gefiel, sahen manche Kritiker Schröders in diesem halbjährigen Aufenthalt den Ursprung der ihm nachgesagten Sympathie für alles Angloamerikanische ${ }^{51}$.

$45 \mathrm{Zu}$ Schröders Kindheit vgl. ebenda, S. 2-5; SCHRÖDER, Wir brauchen eine heile Welt, S. 15; ders., Im Gespräch mit Meinhold Krauss, S. $17 \mathrm{f}$.

$46 \mathrm{Vgl}$. Adelbert SCHRÖDER, Mein Bruder Gerhard Schröder, S. 23.

47 Vgl. ebenda, S. 22. Adelbert Schröder betont dort den großen Einfluß, den der Vater auf das politische Denken seines Bruders hatte.

48 Vgl. ebenda, S. 22; OSTERHELD, Außenpolitik, S. 244.

49 Dies berichtet der damalige Schulfreund Schröders und spätere Professor für Anatomie an der Universität Basel, Gerhard Wolf-Heidegger. Vgl. WOLF-HEIDEGGER, Schulkameradschaft, S. 210. Ein Brief Schröders an seine Familie vom 30. 6. 1930, dem Tag der Räumung des Rheinlandes durch die Alliierten, zeigt, daß er zu dieser Zeit tatsächlich ein sehr negatives Bild von Frankreich hatte. Dort schrieb er unter anderem: „Es muß ein großer Augenblick gewesen sein, als die Trikolore - wie wir hoffen möchten für immer - niedergeholt wurde. Unser Boden ist wieder sauber geworden" Vgl. Adelbert SCHRÖDER, Mein Bruder Gerhard Schröder, S. 37.

$50 \mathrm{Vgl}$. SCHRÖDER, Im Gespräch mit Meinhold Krauss, S. $19 \mathrm{f}$.

51 So z.B. WAGNER, Der neue Mann im Außenamt, S. 27; KOERFER, Kampf ums Kanzleramt, S. 642 f.; SEYDOUX, Beiderseits des Rheins, S. 274. 
Sicherlich könnte man seinen Lebensstil als britisch beeinflußt bezeichnen ${ }^{52}$, doch sollte man nicht vergessen, daß er aus einer friesischen Familie stammte und beide Kulturen durchaus ähnliche Züge aufweisen. Außerdem ist es wohl äußerst gewagt, aus einem Studienaufenthalt von sechs Monaten Dauer unmittelbare Konsequenzen für Schröders außenpolitische Handlungen mehr als 30 Jahre später abzuleiten. Das Sommersemester 1930 verbrachte der angehende Jurist dann in Berlin, bevor er an die Rheinische Friedrich-Wilhelms-Universität nach Bonn wechselte. Dort engagierte sich Schröder in der lokalen Hochschulgruppe der DVP. Er wurde bald ihr Vorsitzender und vertrat sie im Allgemeinen Studentenausschuß. 1932 trat er in Wahlaufrufen, Zeitungsartikeln, Vorträgen und Diskussionen für die Wiederwahl Hindenburgs ein ${ }^{53}$. Noch im selben Jahr meldete er sich zum Examen an, um das Studium noch vor der von ihm und seinem Vater erwarteten Machtübernahme Hitlers zu beenden ${ }^{54}$. Nach sechssemestrigem Studium legte er im Oktober 1932 das erste juristische Staatsexamen mit magna cum laude ab. Anschließend trat er eine erste Referendariatsstellung in Saarburg an.

Zur Jahreswende 1932/33 stand Schröder somit vor dem Einstieg in das Berufsleben. Eigentlich war es seine Absicht, die wissenschaftliche mit einer politischen Laufbahn nach dem Vorbild des Jura-Professors und Reichstagsabgeordneten der DVP, Wilhelm Kahl, zu verbinden ${ }^{55}$. Die geplante Karriere als Politiker mußte er jedoch nach der Machtergreifung Hitlers wegen seiner Mitgliedschaft in der DVP aufgeben. Schröder wollte sich aber zumindest die wissenschaftliche Laufbahn offenhalten und paßte sich daher an die veränderten politischen Gegebenheiten an. Im Frühjahr 1933 wurde ihm eine Assistentenstelle an der juristischen Fakultät in Bonn angeboten, wozu aber die Mitgliedschaft in der NSDAP vorausgesetzt wurde. Er unterschrieb eine Beitrittserklärung ${ }^{56}$. Einige Monate später stellte er einen Aufnahmeantrag in die SA, da, wie sein Bruder berichtet, nach einem Erlaß des zuständigen Oberlandesgerichtspräsidenten Referendare der SA oder SS angehören mußten, um in den Justizdienst übernommen zu werden ${ }^{57}$. Er wurde jedoch schon bald „wegen Interesselosigkeit" von der Liste der Anwärter gestrichen $^{58}$. Obwohl er sich persönlich nichts zuschulden hatte kommen lassen, kritisierte in den fünfziger Jahren die Opposition immer wieder sein damaliges Verhalten. Im Juli 1958 nahmen die Angriffe auf ihn derart zu, daß Schröder im Bundestag eine persönliche Erklärung abgab, in der er die Behauptung strikt zurückwies, er sei ein Anhänger des Nationalsozialismus gewesen: „Ich bin weder vor dem ,Dritten Reich' noch im ,Dritten Reich' auch nur eine Minute lang Nationalsozia-

$52 \mathrm{Vgl}$. KOERFER, Kampf ums Kanzleramt, S. $642 \mathrm{f}$.

53 Vgl. Adelbert SCHRÖDER, Mein Bruder Gerhard Schröder, S. 30-49.

54 Vgl. SCHRÖDER, Jurist, S. 33.

55 Vgl. ebenda.

56 Vgl. Adelbert SCHRÖDER, Mein Bruder Gerhard Schröder, S. 53. Vgl. ferner das Interview mit Gerhard Schröder: „Nazis - A High German Talks“, in: NEwSWEEK vom 1. 2. 1960, S. 30 .

57 Vgl. Adelbert SCHRÖDER, Mein Bruder Gerhard Schröder, S. 58.

58 Vgl. Bulletin 1961, S. 1970. Vgl. ferner „Nazis - A High German Talks“, in: NewsweEK vom 1.2. 1960, S. 30. 
list gewesen. Ich wiederhole: nicht eine Minute lang. "59 Aufgrund intensiver Propaganda aus der DDR kursierte aber weiterhin das Gerücht, er sei ein überzeugter Nationalsozialist gewesen. Als er im Zuge der Regierungsbildung 1961 für das Amt des Außenministers im Gespräch war, sah sich die Bundesregierung schließlich zu einem öffentlichen Dementi gezwungen ${ }^{60}$. Auch seine Doktorarbeit geriet in den Verdacht, sie enthalte nationalsozialistische Inhalte, weil keine deutsche Bibliothek sie in ihrem Bestand führte ${ }^{61}$. Dieser Vorwurf entbehrte jedoch jeder Grundlage: Ende Juli 1933 hatte Schröder sein Doktorexamen mit magna cum laude abgelegt. Seine Promotionsarbeit, die „Die außerordentliche Kündigung von Tarifverträgen" zum Thema hatte, war nicht von nationalsozialistischem Gedankengut beeinflußt. Da am 20. Januar 1934 das Gesetz zur Ordnung der nationalen Arbeit erlassen und damit die Tarifvertragsordnung aufgehoben wurde, hatte die Dissertation ihre Bedeutung verloren. Schröder suchte deshalb um eine Befreiung vom Druckzwang nach, der im Mai 1934 entsprochen wurde. Da durch ein Versäumnis des Dekanats seiner Promotionsakte die Dissertation nicht beigelegt wurde und Schröders eigene Abschrift bei der Ausbombung seiner Anwaltskanzlei im Herbst 1943 in Berlin verbrannte, ist kein Exemplar erhalten ${ }^{62}$.

Im Herbst 1934 zog Schröder nach Berlin, um am Kaiser-Wilhelm-Institut für Ausländisches und Internationales Privatrecht eine Stelle als Assistent anzutreten. Dort legte er im August 1936 das zweite juristische Staatsexamen wiederum mit magna cum laude ab. Angesichts der politischen Lage in Deutschland gab Schröder nun auch den Plan einer wissenschaftlichen Karriere auf. Wie er später erzählte, sah er nur in der Tätigkeit als Anwalt eine Perspektive, „um unter diesen Bedingungen als Jurist ökonomisch und moralisch zu überleben" 63 . Als Assessor trat er in die Anwaltssozietät des Berliner Kammergerichtsanwalts und Notars Dr. Walter Schmidt ein, obwohl mehrere Mitglieder der Kanzlei aus rassischen Gründen verfolgt wurden ${ }^{64}$. Doch mit Schmidt und seiner Praxis war Schröder vertraut, da er bereits als Referendar dort gearbeitet hatte. Mit den Verhältnissen im nationalsozialistischen Deutschland hatte er sich arrangiert. Politisch äußerte er sich nicht mehr, sondern zog sich in die innere Emigration zurück ${ }^{65}$. Schröder blieb jedoch ein engagierter Christ. An der Universität Bonn hatte er regelmäßig die Vorlesungen und Predigten von Karl Barth besucht, dessen theologische Darstellungs- und Überzeugungskraft ihn stark beeinflußten ${ }^{66}$. In Berlin führte ihn sein Weg regelmäßig nach Dahlem, wo Martin Niemöller bis zu seiner Verhaftung

59 BT STENOGRAPHISCHE BERICHTE, Bd. 41, S. 2335.

60 Vgl. dazu Bulletin 1961, S. 1970.

61 Vgl. dazu den Artikel „Quittung vorhanden“, in: Der SPIEgEL vom 24. 2. 1969, S. 36. Vgl. auch HUYN, Die Sackgasse, S. $127 \mathrm{f}$.

62 Vgl. KNÜTEL, Begrüßungsansprache, S. 8.

63 SCHRÖDER, Jurist, S. 33.

64 Die betroffenen Kanzleiangehörigen wurden teils auf Grund ihrer Abstammung, teils auf Grund „nicht-arischer“ Eheschließungen verfolgt. Vgl. dazu BullETiN 1961, S. 1970, sowie den Artikel „Quittung vorhanden“, in: DER SPIEGEL vom 24. 2. 1969, S. 36.

$65 \mathrm{Vgl}$. Adelbert SCHRÖDER, Mein Bruder Gerhard Schröder, S. 58.

66 Vgl. ebenda, S. 50. 
im Jahre 1937 predigte. Schröder sympathisierte mit der Bekennenden Kirche ${ }^{67}$. Über Carl Dietrich von Trotha, einen Freund aus der Referendarzeit und Cousin von Graf Moltke, hatte er Kontakt zum Kreisauer Kreis. Er wurde aber nicht selbst im Widerstand aktiv 68 .

Trotz schlechter Beurteilung durch den NS-Rechtswahrerbund, dem seine Tätigkeit in der Kanzlei Dr. Schmidt, in der inzwischen auch die Tochter Martin Niemöllers beschäftigt wurde ${ }^{69}$, mißfiel, erhielt er am 1. August 1939 die Zulassung als Rechtsanwalt und Fachanwalt für Steuerrecht am Landgericht Berlin. Exakt einen Monat später assoziierte er sich mit der Kanzlei Dr. Schmidt. Zwar erhielt Schröder am 28. August den Einberufungsbescheid in die Wehrmacht, doch konnte er bis zur Zerstörung der Praxis im Herbst 1943 durch einen Bombenangriff seine Anwaltstätigkeit im Rahmen von, zunächst längeren, dann mit dem Kriegsverlauf spärlicher werdenden Arbeitsurlauben fortsetzen ${ }^{70}$. Am 30. Mai 1941 heiratete er Brigitte Landsberg $(* 1917)$, die Schwester eines Freundes aus Bonner Studientagen. Aus der Ehe gingen drei Kinder hervor: Christina ("1941), Jan (*1943) und Antina ("1945). Seine Frau war die Tochter eines Bankiers jüdischer Abstammung. Da sie somit nach NS-Diktion keine ,Vollarierin" war, mußte die Eheschließung genehmigt werden. Das Oberkommando des Heeres erteilte die Erlaubnis unter der Bedingung, daß Schröder auf militärische Beförderungen verzichtete. Bis Kriegsende wurden daher Beförderungsvorschläge von Vorgesetzten mit dem Hinweis auf Schröders "politische Unzuverlässigkeit“ abgelehnt. So befand er sich auch noch 1945 im Rang eines Obergefreiten. Seine seit 1933 andauernde Bemühung um Anpassung an die Parteilinie war mit der Heirat beendet. Sie bedeutete den offenen Bruch mit dem Regime. Noch im gleichen Jahr trat er aus der NSDAP aus ${ }^{71} .1942$ wurde er an der Ostfront bei Cholm verwundet und als nicht mehr fronttauglich in den Heimatdienst versetzt. Nach der Entlassung aus britischer Kriegsgefangenschaft begab er sich im Juni 1945 nach Hamburg, wo seine Eltern sich 1938 im Stadtteil Othmarschen niedergelassen hatten. Schröders Frau hatte gegen Kriegsende bei ihnen mit den Kindern Zuflucht gesucht.

Angesichts der Zerstörung seiner Berliner Rechtsanwaltspraxis stand Schröder bei Kriegsende vor dem beruflichen Nichts. Doch er resignierte nicht. Jetzt sah er die Chance, seinen 1933 begrabenen Traum, politisch tätig zu werden, zu verwirklichen. Wie sein Bruder berichtet, hatte er dieses Vorhaben nie aufgegeben und wollte nun, in der Stunde Null am Neuaufbau der Demokratie in Deutschland mitwirken ${ }^{72}$. Zunächst beabsichtigte Schröder, sich in Hamburg eine neue Existenz aufzubauen. Er plante, entweder wieder als Anwalt zu arbeiten oder in die Stadtverwaltung einzutreten. Dann erfuhr er durch einen Studienfreund, Paul Hübinger, der im Stab des Oberpräsidenten der Nordrhein-Provinz, Hans

\footnotetext{
67 Vgl. ebenda, S. 64-77.

68 Vgl. SCHRÖDER, Im Gespräch mit Meinhold Krauss, S. 25.

69 Vgl. dazu OPPELland, Gerhard Schröder, S. $75 \mathrm{f}$.

70 Vgl. Adelbert SCHRÖDER, Mein Bruder Gerhard Schröder, S. 81-89.

71 Vgl. dazu Bulletin 1961, S. 1970; SCHRÖDER, Im Gespräch mit Meinhold Krauss, S. 21 f.

72 Vgl. Adelbert SCHRÖDER, Mein Bruder Gerhard Schröder, S. $92 \mathrm{f}$.
} 
Fuchs, beschäftigt war, daß in der Verwaltung dieser Provinz Juristen gesucht würden. Da er sich mit Fuchs, der schon von 1922 bis 1933 Oberpräsident war, auf Anhieb verstand - als „Liebe auf den ersten Blick“ beschrieb der sonst so kühle Schröder sein Verhältnis zu dem früheren Reichsminister für die besetzten $\mathrm{Ge}-$ biete - wurde er als Regierungsrat eingestellt und arbeitete als dessen Persönlicher Referent ${ }^{73}$. Damit begann „die erstaunlichste Karriere Nachkriegsdeutschlands"74.

Über Schröders Leben von 1945 bis zu seinem Eintritt in den Bundestag 1949 ist sehr wenig bekannt. Das Wissen über diese vier Jahre beschränkt sich auf wenige Fakten, nämlich, daß er bis 1947 in der Verwaltung der Nordrhein-Provinz tätig war und anschließend als Anwalt für die Treuhandverwaltung der Montanindustrie des Ruhrgebietes arbeitete. Er selbst hat über diesen Lebensabschnitt nie viel berichtet und auch die von seinem Bruder verfaßte Biographie enthält nur spärliche Informationen ${ }^{75}$. Schon 1952 rätselte Die Zeit darüber, warum er 1947 seine Verwaltungslaufbahn plötzlich abbrach und in die Privatwirtschaft wechselte $^{76}$. Nähere Auskunft dazu gibt eine undatierte und unsignierte, maschinenschriftliche Aufzeichnung mit dem Titel „Bundesinnenminister Dr. Gerhard Schröder", die sich im Nachlaß des ehemaligen Ministers für gesamtdeutsche Fragen, Ernst Lemmer, befindet. Der Schlußsatz des Dossiers läßt vermuten, daß es kurz nach dem mysteriösen Verschwinden des Präsidenten des Bundesamts für Verfassungsschutz, Otto John, und seinem Auftauchen in der DDR, also im Jahr 1954, entstand ${ }^{77}$. Bei aller gebotenen Zurückhaltung über den Wahrheitsgehalt dieses Memorandums, das ein weitgehend negatives Bild von Schröder zeichnet und ihn als einen machtgierigen und intriganten Menschen darstellt, soll es dennoch hier referiert werden. Dies geschieht nicht nur, weil es das plötzliche Ausscheiden Schröders aus seiner hohen Position in der Verwaltung erklärt. Das Dossier enthält auch neue Aspekte über die noch immer unklaren Kontakte Konrad Adenauers im Spätsommer 1945 mit den Machthabern der französischen Besatzungszone und bringt mehr Licht in die Hintergründe seiner Entlassung als Kölner Oberbürgermeister Anfang Oktober 1945 durch die britische Besatzungsmacht $^{78}$.

Laut Hans-Peter Schwarz sind bislang sechs Treffen Adenauers mit französischen Offizieren in der Zeit von August bis Oktober 1945 bekannt $^{79}$. Des weiteren hält sich das Gerücht, Adenauer sei außerdem in diesem Zeitraum in der Abtei Maria Laach, die ihm 1933 nach seiner Absetzung als Oberbürgermeister Kölns durch die Nationalsozialisten als Zuflucht diente, mit General de Gaulle zusam-

73 SCHRÖDER, Im Gespräch mit Meinhold Krauss, S. 26.

74 Vgl. den Artikel von Reinhard Appel: „Bundesinnenminister Dr. Gerhard Schröder“, in: STUTTGARTER ZEITUNG vom 20. 1.1954, S. 3.

75 Vgl. dazu Adelbert SCHRÖDER, Mein Bruder Gerhard Schröder, S. 92-96.

76 Vgl. den Artikel „Gerhard Schröder“, in: DiE ZEIT vom 20. 11. 1952, S. 1.

77 ACDP, Nachlaß Lemmer I-280-000/3. Die sechsseitige Aufzeichnung endet bezeichnenderweise mit dem Satz: "Was wußte Dr. John von diesen Vorgängen?“ Zum „Fall John“ vgl. STÖVER, Der Fall Otto John, S. 103-136.

78 Zur Entlassung Adenauers vgl. SCHWARZ, Adenauer I, S. 452-472.

79 Vgl. ebenda, S. 453. 
mengetroffen ${ }^{80}$. Die Aufzeichnung aus dem Nachlaß Lemmers berichtet nun tatsächlich von einem Treffen, das in Maria Laach stattfand. Gesprächspartner Adenauers sei aber nicht der französische Ministerpräsident, sondern der Oberbefehlshaber der französischen Besatzungszone, General Pierre Koenig, gewesen. Außerdem seien Oberpräsident Fuchs, der die Begegnung zusammen mit Adenauer vorbereitet haben soll, ein Adjutant Koenigs sowie ein Vertreter des französischen Außenministeriums namens Michel Leroy-Beaulieu anwesend gewesen. Die Aufzeichnung gibt als Gesprächsziel der beiden deutschen Politiker an, die Kontakte der Nordrhein-Provinz zu denjenigen Regierungsbezirken, die von der Provinz abgetrennt wurden und jetzt unter französischer Kontrolle standen, nicht abreißen zu lassen. Außer den unmittelbar Beteiligten soll von diesem Treffen nur noch der Persönliche Referent des Oberpräsidenten Kenntnis gehabt haben, der als ein Freund von Schröders Vater beschrieben wird, auf dessen Fürsprache Schröder in den engeren Stab von Fuchs gelangte. Dieser, in der Aufzeichnung nicht genannte Referent, bei dem es sich vermutlich um den Studienfreund Hübinger handelt, der Schröder den Posten in der Verwaltung in Düsseldorf verschafft hatte, soll nun Schröder von dem Treffen in Maria Laach erzählt haben. Die Aufzeichnung berichtet ferner, Schröder sei mit seinem Rang als Regierungsrat unzufrieden gewesen und habe von Fuchs verlangt, ihn aufgrund seiner abgeschlossenen juristischen Ausbildung zu befördern. Der Oberpräsident habe dies jedoch mit der Begründung abgelehnt, er solle sich zunächst auf seinem derzeitigen Posten bewähren. Daraufhin habe Schröder enge Kontakte zu dem Stellvertreter von Fuchs und Chef der Abteilung, Allgemeine Verwaltung', dem späteren Bundesinnenminister Robert Lehr, sowie dessen Stellvertreter und späteren Leiter der Staatskanzlei der nordrhein-westfälischen Landesregierung, Hermann Wandersleb, geknüpft, die in Opposition zum Oberpräsidenten standen. Lehr erfuhr jedenfalls von der Konspiration Adenauers und Fuchs' mit den Franzosen und informierte den britischen Militärbefehlshaber der Nordrhein-Provinz, Brigadegeneral John Barraclough. Dieser verfügte nach Rücksprache mit dem Hauptquartier der britischen Militärregierung in Oynhausen den Rücktritt Fuchs' und die Entlassung Adenauers ${ }^{81}$. Zum Nachfolger des abgelösten Oberpräsidenten wurde Lehr bestimmt, während Wandersleb zum Chef der Allgemeinen Verwaltung aufrückte. Als erste Amtshandlung verfügte der neue Oberpräsident die Entfernung des gesamten Mitarbeiterstabes seines Vorgängers. Auffälligerweise entging Schröder nicht nur dieser ,Säuberung', sondern wurde sogar noch zum Oberregierungsrat befördert. Ohne ihn ausdrücklich als Informanten Lehrs zu bezeichnen, unterstellt der Bericht, daß Schröder sein Wissen benutzt habe, um seine Karriere voranzubringen ${ }^{82}$.

$80 \mathrm{Vgl}$. ebenda, S. 464.

81 Fälschlicherweise wird in der hier referierten Aufzeichnung behauptet, beide Entlassungen seien am gleichen Tag erfolgt; Lehr trat jedoch am 2. 10.1945 zurück, während Adenauers Entlassung am 6. 10. 1945 erfolgte. Vgl. dazu ebenda, S. 465 und S. 472.

82 Vgl. die Aufzeichnung "Bundesinnenminister Dr. Gerhard Schröder“, ACDP, Nachlaß Lemmer I-280-000/3. 
Dagegen vertrat Schröder später die Position, Adenauer sei nur deswegen entlassen worden, weil Barraclough mit dem Stand der Aufräumarbeiten in Köln nicht zufrieden war und Adenauers Pläne für den Wiederaufbau der Stadt für übertrieben hielt: „In diesen Vorgang [die Entlassung Adenauers] ist viel hineingeheimnißt worden. Intrigen? Höhere Politik? Ich bin immer noch der Meinung, daß es so simpel war, wie ich es gesagt habe. ${ }^{83}$ Inwieweit Schröder tatsächlich in die Entlassung Adenauers verwickelt war, wird sich wohl kaum mehr aufklären lassen. Tatsache ist, daß er nach dem Wechsel von Fuchs zu Lehr nun einen verhältnismäßig hohen Rang innerhalb der Verwaltung einnahm. Er war mit Wahlund Verfassungsfragen beauftragt und gehörte dem britischen Zonenbeirat in Hamburg als stellvertretendes Mitglied an $^{84}$. Schröders Ziel war aber nicht die Verwaltungslaufbahn. Er verstand sich nicht als Beamter, sondern als Politiker ${ }^{85}$. Jetzt, nach dem Ende der nationalsozialistischen Diktatur, konnte er seinen Lebensplan verwirklichen, im öffentlichen Leben tätig zu werden. Er wollte seine Erfahrungen, seine politischen Vorstellungen und Ideen in den staatlichen Neuaufbau einbringen ${ }^{86}$.

Im Herbst 1946 präsentierte er in der Zeit seine Gedanken über ein künftiges deutsches Wahlrecht. Nach seinen Vorstellungen sollte die Verhältniswahl, wie sie in der Weimarer Republik praktiziert worden war, in einem künftigen deutschen Staatswesen nicht mehr eingeführt werden, da sie die Destabilisierung des politischen Systems fördere. Er plädierte für ein Parlament mit höchstens drei Parteien und favorisierte deshalb das britische Mehrheitswahlrecht ${ }^{87}$. Wohl auch aufgrund dieses Standpunktes wurde er zum Vorsitzenden des Deutschen Wablrechtsausschusses bei der britischen Kontrollkommission berufen. In zahlreichen Zeitungsartikeln bemühte er sich in den folgenden Jahren, die Öffentlichkeit über die Bedeutung des Wahlsystems aufzuklären und von seiner Sicht der Dinge zu überzeugen $^{88}$. Er beteiligte sich deshalb auch an der Gründung der Deutschen Wäblergesellschaft in Frankfurt a.M., die es sich ebenfalls zum Ziel gesetzt hatte, für die Einführung des Mehrheitswahlrechts zu werben ${ }^{89}$. Obwohl die Bundesrepublik im Grunde keine schlechten Erfahrungen damit machte, daß sie sich das britische Wahlrecht nicht zum Vorbild nahm, konnte sich Schröder zeit seines Lebens nicht mit dem personalisierten Verhältniswahlrecht der Bundesrepublik anfreunden. Auch die Einführung der sogenannten Fünf-Prozent-Klausel änderte seine Meinung nicht. Im Gegenteil, er sah es als ein demokratisches Manko an, daß eine kleine Partei wie die FDP die Rolle eines Züngleins an der Waage spielen konnte, wenn weder CDU/CSU noch die SPD über eine absolute Mehrheit der Mandate

\footnotetext{
83 SCHRÖDER, „Donnerwetter, das kann ja fein werden ...“.

$84 \mathrm{Vgl}$. SCHWARZKopf/Wrangel, Chancen, S. 149;. Vgl. dazu auch den Artikel „Gerhard Schröder", in: DIE ZEIT vom 20. 11. 1952, S. 1.

85 Vgl. Adelbert SCHRÖDER, Mein Bruder Gerhard Schröder, S. 95.

86 Vgl. ebenda, S. $92 \mathrm{f}$.

87 Vgl. den Artikel „Zur Kritik des Wahlsystems“, in: DIE ZeIT vom 7. 11. 1946, S. 3. Dieser Beitrag erschien ohne Verfasserangabe. Die Redaktion teilte lediglich mit, es handle sich um den Vorsitzenden des deutschen Wahlrechtsausschusses für die britischen Zone.

$88 \mathrm{Vgl}$. dazu SCHRÖDER, Wir brauchen eine heile Welt, S. 36-43.

89 Vgl. Adelbert SCHRÖDER, Mein Bruder Gerhard Schröder, S. 95.
} 
verfügten, und dadurch weitaus mehr Macht und Einfluß erhielt, als ihrem Stimmenanteil angemessen war. Schröder war der Ansicht, daß die Gründungsmütter und -väter der Bundesrepublik in diesem Punkt die Weichen falsch gestellt hät$\operatorname{ten}^{90}$.

Noch ist die Frage aber nicht beantwortet, warum er trotz seines raschen Aufstiegs in der Verwaltung der Nordrhein-Provinz 1947 schließlich den Dienst quittierte. Die erwähnte Aufzeichnung aus dem Nachlaß von Ernst Lemmer, die inhaltlich im übrigen in bemerkenswerter Weise mit einem Artikel im Spiegel aus dem Jahr 1959 übereinstimmt ${ }^{91}$, gibt folgende Erklärung: Das zunächst gute Verhältnis zwischen Schröder und Lehr habe sich sehr rasch abgekühlt, weil Lehr feststellen mußte, daß aus seinem Büro Informationen an die britische Militärregierung weitergegeben wurden. Der Verdacht des Oberpräsidenten fiel sehr rasch auf seinen früheren Verbündeten, unterhielt dieser doch ein Verhältnis mit der Sekretärin Lehrs. Da sich Schröder nicht an die Anweisung hielt, die Beziehung abzubrechen, beabsichtigte Lehr, ein Diziplinarverfahren einzuleiten, um ihn aus dem Verwaltungsdienst zu entfernen. Wandersleb riet ihm aber von diesem Schritt $a b$, weil er damit rechnete, daß die Militärregierung ihre Zustimmung nicht erteilen würde, falls Schröder tatsächlich ein Informant der Briten sei. Daraufhin entschloß man sich, ihn auf einen unwichtigen Posten abzuschieben. Auch als Lehr nach der Gründung des Landes Nordrhein-Westfalen aus dem Amt geschieden und Schröder jetzt unter Innenminister Menzel in der Regierung Rudolf Amelunxen tätig war, führte er sein außereheliches Verhältnis fort. Eine Urlaubsreise an den Tegernsee, bei der er seine Begleiterin als seine Ehefrau ausgab, führte jedoch zu polizeilichen Ermittlungen, bei denen unter anderem auch seine Frau Brigitte in Hamburg vernommen wurde. Da die Polizei auch die nordrhein-westfälische Regierung von diesem Vorfall informierte, gab Schröder dem Dossier zufolge seine Beamtentätigkeit auf92. Anschließend ließ er sich als freier Anwalt in Düsseldorf nieder ${ }^{93}$. Kurz darauf empfahl ihn der spätere Ministerpräsident von Nordrhein-Westfalen, Karl Arnold, dem Präsidenten der Stahltreuhändervereinigung, Heinrich Dinkelbach. Schröder war nun hauptsächlich als Abteilungsleiter für diese Behörde tätig, die an der Entflechtung der Stahlindustrie mitwirkte. In dieser Funktion stand er dem Aufsichtsrat über die Hüttenwerke Ruhrort-Meidrich

90 Vgl. SCHRÖDER, Jurist, S. 34; Adelbert SCHRÖDER, Mein Bruder Gerhard Schröder, S. 96.

91 Vgl. den Artikel "Der Mitschreiber“, in: DER SPIEGEL vom 22. 7. 1959, S. 16-25. Schröder reagierte auf diese Veröffentlichung sehr erbost. Am 31.7. 1959 schrieb er an Adenauer, man könne „im Interesse der Pressefreiheit nur wünschen, daß sonst bald DDT auch gegen solche Publikationen erfunden wird. Im übrigen enthält der Aufsatz über mich nicht weniger als 75 (gezählte!) Unrichtigkeiten." Für das Schreiben vgl. StBKAH III/24. Selbst einer der schärfsten Kritiker Schröders, Wolfgang Wagner, meinte später, es habe sich um eine „ausgesprochen böswillige Titelgeschichte“ gehandelt. Vgl. WAGNER, Der neue Mann im Außenamt, S. 23.

92 Vgl. die Aufzeichnung „Bundesinnenminister Dr. Gerhard Schröder“, ACDP, Nachlaß Lemmer I-208-000/3, S. 4-6. Zu seinem Ausscheiden aus der Verwaltung teilte Schröder rückblickend lediglich mit, er habe sich in der Regierung Amelunxen nicht mehr wohlgefühlt. Vgl. SCHRÖDER, Im Gespräch mit Meinhold Krauss, S. 27.

$93 \mathrm{Vgl}$. GOYKE, Die 100 von Bonn, S. 245. 
und Haspe vor. Er erhielt so einen Einblick in die vielfältigen Probleme der Montanindustrie ${ }^{94}$, geriet aber in den Ruf, ein Mann der Industrie zu sein ${ }^{95}$.

Schröder gehörte zu den Gründungsmitgliedern der CDU im Rheinland. 1947 fiel er Adenauer, damals Vorsitzender der CDU in der britischen Zone, erstmals auf, als Schröder, noch als Referent in der Verwaltung Nordrhein-Westfalens tätig, eine für die CDU günstige Wahlkreiseinteilung bei den ersten Landtagswahlen vornahm ${ }^{96}$. Zwei Jahre später wurde er als Bundestagskandidat im Wahlkreis Düsseldorf-Mettmann aufgestellt, obwohl Adenauer eigentlich einen anderen Kandidaten ins Auge gefaßt hatte. Schröder sollte seiner Ansicht nach erst im Landtag parlamentarische Erfahrung sammeln: „Der Schröder ist zu jung, der sollte in den Landtag gehen, der kann sich ja unter den alten Füchsen in so einer Riesenfraktion nicht durchsetzen. "97 Im Wahlkampf versuchte Schröder die Bürger zu überzeugen, daß er für einen politischen Neuanfang steht: „Ich bin kein alter Parlamentarier und kein Routinier der alten Schule" - mit diesen Worten begann sein Wahlaufruf ${ }^{98}$. Mit klaren Aussagen und dem Einsatz moderner Wahlkampfmethoden gelang es ihm, den Wahlkreis, in dem eigentlich nach dem Ausgang der Landtagswahlen 1947 ein Sieg der SPD erwartet wurde, mit einem Vorsprung von 3.000 Stimmen zu gewinnen ${ }^{99}$. So zog er im September 1949 als weithin unbekannter parlamentarischer Neuling in den ersten Deutschen Bundestag ein.

Für die Öffentlichkeit, aber auch für weite Kreise innerhalb der CDU/CSU war er ein noch unbeschriebenes Blatt. Weder übte er bislang ein Parteiamt aus, noch verfügte er über eine Hausmacht, auf die er sich stützen konnte. Dennoch rechnete er allen Ernstes damit, Adenauer würde ihm ein Ministeramt antragen ${ }^{100}$. Als sich diese Hoffnung nicht erfüllte, konzentrierte er sich auf den Aufstieg in der Fraktion. Er ließ sich nicht auf die Hinterbänke des Parlaments abschieben, sondern verstand es sehr schnell, sich einen Namen zu machen. Aufgrund seiner Tätigkeit in der Montanindustrie war er ein juristischer Experte in diesem Bereich. In seiner parlamentarischen Arbeit konzentrierte er sich daher zunächst auf die Neuordnung der Kohle- und Stahlindustrie. Besonders intensiv widmete er sich dem Problem der Mitbestimmung der Arbeitnehmer in der Montanindustrie und setzte sich nachdrücklich für eine paritätische Regelung ein. In Zusammenarbeit mit dem Vorsitzenden des CDU-Bundesausschusses für Wirtschaftspolitik, Franz Etzel, bereitete er in der Unionsfraktion die entsprechenden Bestimmungen

94 Vgl. SChwarZ, Dr. Gerhard Schröder, S. 17, sowie den Artikel von Reinhard Appel: „Bundesinnenminister Dr. Gerhard Schröder“, in: Stuttgarter ZeItUNG vom 20. 1. 1954, S. 3.

95 Vgl. Oppelland, Gerhard Schröder, S. 77.

96 Vgl. den Artikel von Reinhard Appel: „Bundesinnenminister Dr. Gerhard Schröder“, in: STUTTGARTER ZEITUNG vom 20. 1. 1954, S. 3.

97 Zitiert nach dem Artikel „Gerhard Schröder“, in: DIE ZeIT vom 20. 11. 1952, S. 1. Vgl. ferner SCHRÖDER, „Donnerwetter, das kann ja fein werden ...“.

98 SCHRÖDER, Wir brauchen eine heile Welt, S. 52 (Hervorhebungen im Original).

99 Vgl. Adelbert SCHRÖDER, Mein Bruder Gerhard Schröder, S. 99; SCHWARZ, Dr. Gerhard Schröder, S. 19.

100 Vgl. SCHRÖDER, „Donnerwetter, das kann ja fein werden ...“. 
vor ${ }^{101}$. Schröder wird deswegen zuweilen als „einer der Väter der Sozialpartnerschaft" bezeichnet ${ }^{102}$. Den Widerstand mancher Teile der Industrie - die „Kräfte der trägen Beharrung“, wie er sie nannte - gegen eine paritätische Mitbestimmung der Arbeitnehmer, wies er am 27. Juli 1950 im Bundestag zurück und argumentierte, „daß nur in einer Überwindung der Verhältnisse, wie wir sie gehabt haben, eine Aussicht auf Zukunft gegeben ist" ${ }^{103}$. Im Rückblick sah auch Schröder selbst sein Engagement in dieser Frage, das ihn einer breiten Öffentlichkeit bekannt machte, als den eigentlichen Auftakt seiner politischen Karriere an ${ }^{104}$. Gleichzeitig arbeitete er sich in der Unionsfraktion nach oben. Schon 1951 wurde er zum Stellvertreter des Fraktionsvorsitzenden der CDU/CSU, Heinrich von Brentano, berufen. Auch in der Parteihierarchie stieg er rasch auf. Er war jetzt Mitglied des Landesvorstands der CDU Nordrhein-Westfalen sowie des Bundesvorstands. Schröder gehörte zu den „hoffnungsvollen jungen Männern“ der Partei105.

Seine wesentliche Aufgabe als Politiker sah er in den Anfangsjahren der Bundesrepublik, als diese noch ungefestigt erschien und sich ihre politische Stabilität erst noch erweisen mußte, darin, der Demokratie ein sicheres Fundament zu errichten. Unentwegt warnte er vor einem Rückfall in die Krisen der Weimarer Zeit. Die erste deutsche Republik war für ihn der Inbegriff eines instabilen Staatsgebildes, welches ein politisches Klima zuließ, das letztendlich den Aufstieg und die Herrschaft des Nationalsozialismus ermöglicht hatte. Sein Wirken war daher darauf gerichtet, die Organe und Institutionen des neuen Staates in der Gesellschaft zu verankern, den Rechtsstaat zu festigen und ihm in allen Bereichen des öffentlichen Lebens Geltung zu verschaffen. Dies erschien ihm um so wichtiger, da die Bundesrepublik als künstliches Produkt der Besatzungszeit, deren staatliche Autorität zudem nur in einem Teil Deutschlands galt, die Hypothek trug, sich Ansehen und Legitimation beim Bürger erst erwerben zu müssen. Sie durfte auf keine natürliche Verbundenheit des Einzelnen mit dem Staat bauen, sondern mußte selbst die Voraussetzungen schaffen, daß sich ein Staatsbewußtsein in der Bevölkerung allmählich entwickeln konnte ${ }^{106}$.

Die Ernennung zum Innenminister im zweiten Kabinett Adenauer am 20. Oktober 1953 als Nachfolger seines früheren Düsseldorfer Chefs, Robert Lehr, war für die politischen Kreise in der Bundesrepublik keine Überraschung mehr, obwohl Schröder in der ersten Legislaturperiode nicht gerade als Gefolgsmann Adenauers aufgetreten war. Die Personalentscheidung Adenauers fand auch in der Fraktion einhellige Zustimmung ${ }^{107}$. Schröder war nun Mitglied der Bundesregierung - "glücklicherweise“, so sein späteres Urteil, vier Jahre später, als er eigentlich erhofft hatte ${ }^{108}$. Die mit dem neuen Amt verbundene, gesteigerte öffentliche

101 Vgl. WENZEL, Gerhard Schröder, S. $173 \mathrm{f}$.

102 So Meinhold Krauss in SCHRÖDER, Im Gespräch mit Meinhold Krauss, S. 28.

103 BT STENOGRAPHISCHE BERICHTE, Bd. 4, S. 2937.

104 Vgl. SCHRÖDER, Im Gespräch mit Meinhold Krauss, S. 30.

105 ECKARDT, Ein unordentliches Leben, S. 276.

106 Vgl. dazu die Auswahl von entsprechenden Artikeln und Reden Schröders aus dieser Zeit in SCHRÖDER, Wir brauchen eine heile Welt, S. 44-47 und S. 56-111.

107 Vgl. SCHWARZ, Dr. Gerhard Schröder, S. 19.

108 SCHRÖDER, „Donnerwetter, das kann ja fein werden ...“. 
Aufmerksamkeit nützte er, um seine politischen Überzeugungen der Öffentlichkeit darzulegen. In seinen Ansprachen hob er vor allem den positiven Unterschied zwischen der jetzigen Demokratie westlicher Prägung und dem deutschen Obrigkeitsstaat früherer Zeit hervor. In seinen öffentlichen Reden wandte er sich oft direkt an den einfachen Bürger, den „Mann auf der Straße“, wie ihn Schröder bezeichnete, der für ihn der Garant der Demokratie war. Ihn rief er dazu auf, sich nicht länger als Untertan des Staats zu fühlen, sondern als ein Teil desselben, nämlich als Staatsbürger, der die staatlichen Entscheidungen mitbestimmt. Schröder hob jedoch nicht nur die Verantwortung des Einzelnen für die Etablierung einer gefestigten Demokratie hervor, sondern stellte auch die seines Erachtens entscheidende Rolle der Presse für die Entstehung einer positiven Grundeinstellung im Volk gegenüber dem noch jungen Staatsgebilde heraus ${ }^{109}$.

Natürlich war ihm bewußt, daß Appelle allein nicht genügten, obrigkeitsstaatliches Denken und undemokratische Traditionen zurückzudrängen. Als Innenminister förderte er deshalb den Auf- und Ausbau der politischen Bildung nach Kräften. Die Stabilität der Bundesrepublik hing aber für ihn nicht nur von der Solidarität ab, die der Bürger für den Staat empfand. Ebenso entscheidend erachtete er die Aufrechterhaltung der inneren und äußeren Sicherheit. Seine Warnungen vor kommunistischen Unterwanderungen und Umsturzplänen, seine Forderung nach einer Luftschutzplanung zum Schutz der Zivilbevölkerung bei einem bewaffneten Angriff sowie seine Pläne für eine Notstandsgesetzgebung stießen jedoch teilweise auf erbitterten Widerstand bei der parlamentarischen Opposition. Hatten die Sozialdemokraten bei seinen Reden im Bundestag zur Mitbestimmungsfrage noch applaudiert, sahen sie jetzt in ihm den "Schwarzen Mann" des Kabinetts. Schröder geriet in den Ruf, er sei ein Anhänger einer autoritären Staatsideologie ${ }^{110}$. Er wurde zu einem der „prominentesten Prügelknaben von Bonn“, der „im Bundestag mit schöner Regelmäßigkeit Tumulte auslöste und von den Sozialdemokraten mit den bösartigsten Zwischenrufen geradezu überschüttet“ wurde ${ }^{111}$. Er ließ sich jedoch nicht von seinem Weg abbringen, da er überzeugt war, eine wehrhafte Demokratie müsse sowohl auf dem rechten, als auch auf dem linken Auge wachsam bleiben ${ }^{112}$. So führte er auch die beiden bisher einzigen Parteiverbotsprozesse in der Geschichte der Bundesrepublik beim Bundesverfassungsgericht durch. Schröder hatte die Verfahren gegen die KPD und die rechtsradikale Sozialistische Reichspartei (SRP) noch von seinem Vorgänger übernommen. Den Prozeß gegen die KPD - dies behauptete er zumindest später - hätte er aber gerne eingestellt, da die Behörden seiner Ansicht nach auch ohne Verbot dieses Problem in den Griff bekommen hätten ${ }^{113} .1960$ mußte der Innenminister die von ihm eingebrachte Gesetzesinitiative für eine Notstandsregelung zurückziehen, da für die weitreichenden Rechte, die die Vorlage der Exekutive im Ernstfall

109 Vgl. SCHRÖDER, Wir brauchen eine heile Welt, S. 83-106.

110 Ebenda, S. 81.

111 HoFf, Demokratie und Autorität, S. 42.

112 Vgl. SCHRÖDER, Wir brauchen eine heile Welt, S. 145-149 und S. 180-182.

113 Vgl. SCHRÖDER, Eine Schlacht um Otto John; ders., Im Gespräch mit Meinhold Krauss, S. 37. 
gewährte, keine parlamentarische Zwei-Drittel-Mehrheit zu finden war. Zu Zugeständnissen an die Opposition war Schröder nicht bereit: „Besser keine, als eine schlechte Notstandsregelung “, meinte er trocken ${ }^{114}$. Mit den im Mai 1968 durch die Große Koalition beschlossenen Notstandsgesetzen konnte er sich deswegen nie anfreunden. Schröder hielt sie nicht für praktikabel, da sie die Rechte der Bundesregierung seiner Meinung nach zu stark einschränkten. Er war der Ansicht, die Regierung müßte im Ernstfall freie Hand haben: „Der Notstand ist die Stunde der Exekutive."115

Die Rücknahme der von ihm ausgearbeiteten Notstandsregelung blieb nicht die einzige politische Niederlage Schröders in seiner Zeit als Innenminister. So scheiterte er 1961 mit Adenauers Plänen für ein von der Bundesregierung betriebenes Deutschland-Fernsehen am Bundesverfassungsgericht. Die schwerste Amtskrise hatte er jedoch bereits 1954 nur wenige Monate nach seinem Amtsantritt zu überstehen, als der Präsident des Verfassungsschutzes, Otto John, am 20. Juli auf mysteriöse Weise verschwand und wenig später in Ost-Berlin wieder auftauchte. Die Opposition forderte den Rücktritt des Innenministers, da er für den Verfassungsschutz verantwortlich war. Obwohl Schröder im Verlauf dieser ersten großen politischen Bewährungsprobe einige Male ungeschickt handelte - beispielsweise wurde seine Idee heftig kritisiert, für die Aufklärung der Affäre eine halbe Million DM als Belohnung auszuschreiben ${ }^{116}$-, konnte er im Bundestag den gegen ihn erhobenen Vorwurf entkräften, er habe seine Aufsichtspflicht gegenüber John verletzt und sich auf dem Schleudersitz des Innenministers behaupten ${ }^{117}$.

Die acht Jahre, die er insgesamt dem Bundesministerium des Inneren vorstand, wurden bis zum heutigen Tag von keinem seiner Nachfolger übertroffen. Aufgrund der weitgehend reibungsfreien Zusammenarbeit in dieser Zeit mit dem Kanzler bekam Schröder das Image eines „Musterknabe[n] des Kabinetts“118. Er selbst verglich seine Beziehung zu Adenauer mit einem „Vater-Sohn-Verhältnis" ${ }^{119}$. Kritiker warfen ihm vor, zu wenig innovativ zu sein. Der Journalist Wolfgang Wagner meinte: „Für ihn war die Parole Keine Experimente nie ein bloßer Wahlslogan, sondern ein selbstverständliches Prinzip des Handelns."120 Insgesamt gesehen kann Schröders Amtsführung aber als erfolgreich bezeichnet werden. Sie verschaffte ihm Reputation sowohl innerhalb der Partei, als auch in der Öffentlichkeit. Sein Amt als Vorsitzender des Evangelischen Arbeitskreises der Union, das er als Nachfolger von Hermann Ehlers und Robert Tillmanns seit 1955 bekleidete, machte ihn zudem zum Sprecher des protestantischen Flügels von

114 Adelbert SCHRÖDER, Mein Bruder Gerhard Schröder, S. 41. Vgl. auch SCHRÖder, Wir brauchen eine heile Welt, S. 164-180.

115 SCHRÖDER, Im Gespräch mit Meinhold Krauss, S. 34. Vgl. auch ders., Eine Schlacht um Otto John.

116 Vgl. SCHRÖDER, Eine Schlacht um Otto John; Adelbert SCHRÖDER, Mein Bruder Gerhard Schröder, S. 108.

117 Vgl. BT StenOGRAPHische Berichte, Bd. 21, S. 1953-1959; SCHrÖDER, Eine Schlacht um Otto John.

118 WAGNER, Der neue Mann im Außenamt, S. 23.

119 SCHRÖDER, "Donnerwetter, das kann ja fein werden ...".

120 WAGNER, Außenamt, S. 26 (Hervorhebung im Original). 
CDU und CSU. Die Treffen des Arbeitskreises nutzte er oftmals, um auf die Bedeutung eines christlich-moralischen Fundaments jeglicher Politik hinzuwei$\operatorname{sen}^{121}$.

Schon Ende der fünfziger Jahre wurde er in Politiker- und Journalistenkreisen als ein potentieller Nachfolger Adenauers gehandelt. Eine Umfrage des Meinungsforschungsinstituts Emnid vom Januar 1958 zeigt aber, daß die Bevölkerung den Innenminister noch nicht zum unmittelbaren Kreis der Kronprinzen zählte: Nur 3\% der Befragten nannten auf die Frage, wer ihrer Meinung nach Adenauer als Kanzler beerben sollte, Schröder. Hingegen plädierten $28 \%$ für von Brentano, weitere $30 \%$ wünschten sich Erhard als Bundeskanzler ${ }^{122}$. Er selbst hatte keine Zweifel an seiner Befähigung zu höheren Aufgaben: „Ein Politiker von meinem Format kann nur noch zwei Ambitionen haben: Außenminister oder Kanzler“, soll er schon 1959 geäußert haben ${ }^{123}$. Auch Adenauer spürte schon frühzeitig, daß Schröder nach Höherem strebte: „Wissen se, wat der will? Der will auf dem Sessel sitzen", und zeigte auf seinen eigenen ${ }^{124}$. Daß sein Ehrgeiz tatsächlich auf den Kanzlerstuhl zielte, zeigt sein Verhalten in den Jahren 1958/59, als ein Nachfolger für den scheidenden Bundespräsidenten Heuss gesucht wurde. Hier betrieb er Politik in eigener Sache und bemühte sich sehr geschickt, seinen Mitbewerber um die Kanzlernachfolge, Wirtschaftsminister Erhard, auf den Posten des Bundespräsidenten abzuschieben. Schröder begann bereits im Oktober 1958, mögliche Kandidaten für die Nachfolge von Heuss ins Auge zu fassen ${ }^{125}$. Anfang 1959 versuchte er dann, Erhard zur Kandidatur zu überreden. Schröder köderte ihn mit dem Hinweis, er könne, sobald Adenauer abtrete, jederzeit das Präsidentenamt wieder aufgeben, um selbst Kanzler zu werden ${ }^{126}$. Auf diese Weise versuchte er, den aussichtsreichsten Konkurrenten um das Amt des Bundeskanzlers wegzuloben ${ }^{127}$. Der weitere Verlauf der "Präsidentschaftskrise“ ist bekannt und braucht hier nur in aller Kürze geschildert werden: Erhard erklärte sich zunächst zur Kandidatur bereit. Plötzlich entschloß sich Adenauer aber, selbst das Amt zu übernehmen. Schließlich zog er jedoch seine Bewerbung wieder zurück, worauf die Union wegen dieser Kehrtwende heftige Kritik an ihrem Vorsitzenden übte ${ }^{128}$.

$121 \mathrm{Zu}$ der christlichen Fundierung von Schröders Politikauffassung, vgl. die Ansprachen von Bundeskanzler Helmut Kohl, Bundestagspräsidentin Rita Süssmuth und Bischof a. D. Hermann Kunst anläßlich des Staatsaktes für Gerhard Schröder im Bundestag, in: Bulletin 1990, S. 41-44. Vgl. ferner Adelbert SCHRÖDER, Mein Bruder Gerhard Schröder, S 116-119.

122 Vgl. dazu KoERFER, Kampf ums Kanzleramt, S. 181.

123 Zitiert nach GoYke, Die 100 von Bonn, S. 246.

124 Zitiert nach dem Artikel „Gerhard Schröder“, in: DIE ZEIT vom 20. 11. 1952, S. 1 (Hervorhebung im Original).

$125 \mathrm{Vgl}$. HeuSs, Tagebuchbriefe, S. 347.

126 Vgl. WAGNER, Die Bundespräsidentenwahl 1959, S. 14f. Zu Schröders Drängen, Erhard als Bundespräsidenten zu nominieren, vgl. auch KRONE, Tagebücher, Eintrag vom 3. 2. 1959, S. 329.

127 So bereits der Eindruck Heinrich Krones: KRONE, Tagebücher, Einträge vom 26. 2. und 1. 3. 1959, S. 335 f. Vgl. ferner CONZE, Die gaullistische Herausforderung, S. 139; KoERFER, Kampf ums Kanzleramt, S. 236;.

128 Vgl. dazu WAGNER, Die Bundespräsidentenwahl 1959, S. 13-72. 
Einer der wenigen, die Adenauer verteidigten, war Schröder. Als Adenauer in der Fraktionssitzung der Union am 5. Juni 1959 unter schweren Beschuß geriet, stellte sich der Innenminister auf die Seite des angeschlagenen Kanzlers ${ }^{129}$. In der Haushaltsdebatte des Bundestags am 11. Juni 1959 nahm er ihn erneut in Schutz, indem er Adenauers Aussage bestätigte, seine Entscheidung, die Kandidatur niederzulegen, sei nicht spontan erfolgt. Schröder erinnerte daran, daß dieser bereits am 14. Mai 1959 mitgeteilt hatte, er sei zu 90\% entschlossen, nicht zu kandidieren. Er unterschlug dabei aber, daß der Kanzler in den Wochen danach seine Bewerbung um so nachhaltiger bekräftigt hatte ${ }^{130}$. Schröder bestätigte durch seine Unterstützung Adenauers seinen Ruf als treuer Paladin. Durch sein loyales Verhalten stieg er in dessen Wertschätzung ${ }^{131}$. In realistischer Einschätzung der politischen Machtverhältnisse in CDU und CSU konzentrierte sich Schröder aber nicht auf die Nachfolge Adenauers, sondern zunächst auf die Übernahme des Auswärtigen Amts und machte aus diesem Ziel auch keinen Hehl132. Seine heimliche Leidenschaft galt nämlich schon seit langem der Außenpolitik. Auffallend oft hatte er sich bereits als Bundestagsabgeordneter in Debatten oder in Zeitungskolumnen zu Fragen der auswärtigen Politik geäußert ${ }^{133}$. Als Innenminister ließ er sich vom Regierungssprecher, Felix von Eckardt, regelmäßig über die aktuelle außenpolitische Lage informieren ${ }^{134}$. Auf diese Weise bemühte er sich, seine mangelnde Erfahrung auszugleichen. So gut es ging, bereitete er sich auf den Moment vor, in dem der Ruf ins Auswärtige Amt erklingen würde. Da er geschickterweise seine Ambition nie verborgen hatte, konnte er damit rechnen, im Falle einer Neubesetzung dieses Postens zum ersten Kreis der Anwärter zu gehören.

129 Vgl. KOERfER, Kampf ums Kanzleramt, S. 320-324; KrONE, Tagebücher, Eintrag vom 5.6. 1959, S. $360 \mathrm{f}$.

130 Vgl. KOERFER, Kampf ums Kanzleramt, S. 341-344.

131 Vgl. SCHWARZ, Adenauer II, S. 170 und S. $357 \mathrm{f}$.

132 Vgl. SCHRÖDER, Im Gespräch mit Meinhold Krauss, S. 32; BLANKENHORN, Verständnis, S. 290; ECKARDT, Ein unordentliches Leben, S. 276; KRONE, Tagebücher, Einträge vom 31. 1. und 3. 2. 1953 sowie vom 12. 6. 1958, S. 112 f. bzw. S. 304.

133 Vgl. SCHRÖDER, Wir brauchen eine heile Welt, S. 65-77.

$134 \mathrm{Vgl}$. ECKARDT, Ein unordentliches Leben, S. 276. 